\title{
BVI photometry and the spectroscopy of Nova Scuti 2005 N.2 ${ }^{\star}$ (Research Note)
}

\author{
U. Munari ${ }^{1}$, A. Siviero ${ }^{1}$, H. Navasardyan ${ }^{1}$, and S. Dallaporta ${ }^{2}$ \\ 1 INAF Osservatorio Astronomico di Padova, via dell'Osservatorio 8, 36012 Asiago (VI), Italy \\ e-mail: munari@pd.astro.it \\ 2 via Filzi 9, 38034 Cembra (TN), Italy
}

Received 19 December 2005 / Accepted 7 March 2006

\section{ABSTRACT}

\begin{abstract}
Our CCD photometry of Nova Scuti 2005 N.2 (=V477 Sct) shows it to be a very fast nova, which is characterized by $t_{2}=3$ and $t_{3}=$ 6 days, affected by a $E_{B-V} \geq 1.3$ mag reddening, and which peaked at $V \sim 9.8$ mag on $\sim$ Oct. 12.0 UT. The nova was probably entering a dust condensation episode or brightness oscillations during the transition phase when it became unobservable for the seasonal conjunction with the Sun. Absolute spectrophotometry shows it to belong to the He/N class. The emission line width at half intensity is $2600 \mathrm{~km} \mathrm{~s}^{-1}$. At least five ripples are identified in the high resolution emission lines profiles at radial velocities ranging from -980 to $+700 \mathrm{~km} \mathrm{~s}^{-1}$. The nova erupted at a large distance from the Sun and at an appreciable height above the Galactic plane, suggesting an association with the Galactic bulge (unusual for a $\mathrm{He} / \mathrm{N}$ nova). The progenitor was too faint to be recorded on DSS1/2 survey plates, when setting the outburst amplitude to $\Delta V \geq 11 \mathrm{mag}$.
\end{abstract}

Key words. stars: novae, cataclysmic variables

\section{Introduction}

Nova Scuti 2005 N.2 (=V477 Sct) was discovered by Pojmanski (2005) on $\mathrm{ASAS}^{1}$ patrol images, shining at $V=12.0$ on Oct. 11.026 and at $V=10.4$ on Oct. 13.066 (UT) indicating that the nova was first caught during the rise to maximum. The nova was independently discovered by Haseda (2005). An accurate astrometric position was derived by Puckett (2005) as $\alpha=$ $183842.93, \delta=-121615.6$ (corresponding to galactic coordinates $l=20.57, b=-2.79$ ). No field star is visible at this position on DSS1 and DSS2 survey plates, indicating an outburst amplitude $\Delta V \geq 11 \mathrm{mag}$. The absence of the progenitor on the 2MASS survey excludes its belonging to the class of recurrent novae with a cool giant donor star (like T CrB or RS Oph).

Very little is known about this nova that was discovered shortly before becoming lost in the seasonal conjunction with the Sun. Das et al. (2005) report that on Oct. 15.75 (UT) the nova displayed prominent $\mathrm{H}$ I emission lines of the Paschen and Brackett series on infrared spectra (1.08-2.35 $\mu \mathrm{m}$ range), indicating an FWZI of $6000 \mathrm{~km} \mathrm{~s}^{-1}$, while an optical spectrum on 16.43 (UT) by Fujii (2005) shows a reddish continuum with broad emission lines including $\mathrm{H} \alpha, \mathrm{H} \beta$, and O I $7773 \AA$ characterized by an $F W H M$ of $2900 \mathrm{~km} \mathrm{~s}^{-1}$.

\section{Observations}

Low and medium resolution spectra of Nova Scuti 2005 N.2 were secured on Oct. 27.7 with the AFOSC imager+spectrograph mounted on the $1.82 \mathrm{~m}$ telescope operated

\footnotetext{
* Spectra are available in electronic form at the CDS via anonymous ftp to cdsarc.u-strasbg.fr (130.79.128.5) or via http://cdsweb.u-strasbg.fr/cgi-bin/qcat?]/A+A/452/567

${ }^{1}$ http://archive.princeton. edu/ asas/
}

in Asiago by INAF Astronomical Observatory of Padova. We obtained absolutely fluxed low-resolution spectrophotometry covering the range $3505-7815 \AA$ with a dispersion of $4.2 \AA /$ pix. The spectrum is presented in Fig. 1, with line identification superimposed. Higher resolution emission line profiles were obtained with holographic grisms over short wavelength intervals covering $\mathrm{H} \alpha$ and OI $8447 \AA$ lines (6390-7045 $\AA$ at $0.6 \AA /$ pix, and 8265-9165 $\AA$ at $0.9 \AA /$ pix, respectively). The velocity profile of both lines is presented and compared in Fig. 2. The spectra can be obtained in electronic form from http://ulisse.pd.astro.it/novasct2005n2/ and from the CDS.

The CCD $B, V, I_{\mathrm{C}}$ photometry on Nova Scuti 2005 N.2 was secured from a private observatory near Cembra (Trento), Italy, housing a $28 \mathrm{~cm}$ Schmidt-Cassegrain telescope. The data reduction was performed in a standard fashion in IRAF. The photometric data are reported in Table 1, and the photometric evolution of the nova is presented in Fig. 3. The photometric data are calibrated on nearby TYC 5700-812-1 used as a comparison star (being present in the same frames as the nova) for which we adopted $B_{\mathrm{J}}=10.96, V_{\mathrm{J}}=10.27, I_{\mathrm{C}}=9.50$. Johnson's $B_{\mathrm{J}}$ and $V_{\mathrm{J}}$ are derived from Tycho-2's $B_{\mathrm{T}}$ and $V_{\mathrm{T}}$ following Bessell (2000) transformations. $I_{\mathrm{C}}$ is derived from Johnson's $B_{\mathrm{J}}$ and $V_{\mathrm{J}}$ following Caldwell et al. (1993) transformations.

\section{Photometric evolution}

The lightcurve in Fig. 3 is clearly that of a very fast nova. The exact maximum is somewhat uncertain, there being no data between Oct. 11.026, when the nova was discovered on the rising branch at $V=12.0$, and Oct. 13.066 when it was at $V=10.4$ and fading already. A reasonable hand-drawn fit of the early light-curve in Fig. 3 suggests that the maximum was reached 


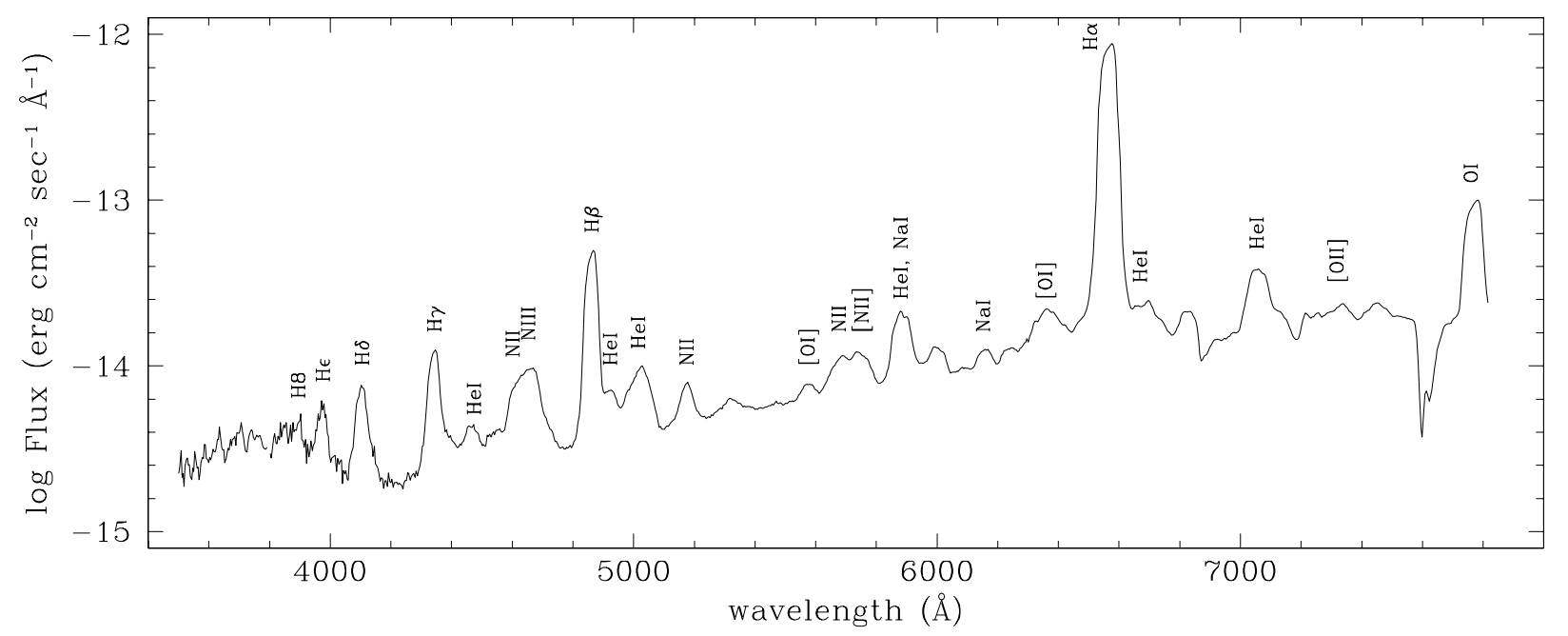

Fig. 1. The absolutely fluxed 3500-7800 Å low resolution spectrum of Nova Scuti 2005 N.2 observed on 2005 Oct. 27.76 UT. Most probable identification for the emission lines is given. The ordinate scale is logarithmic to emphasize the visibility of weaker features.

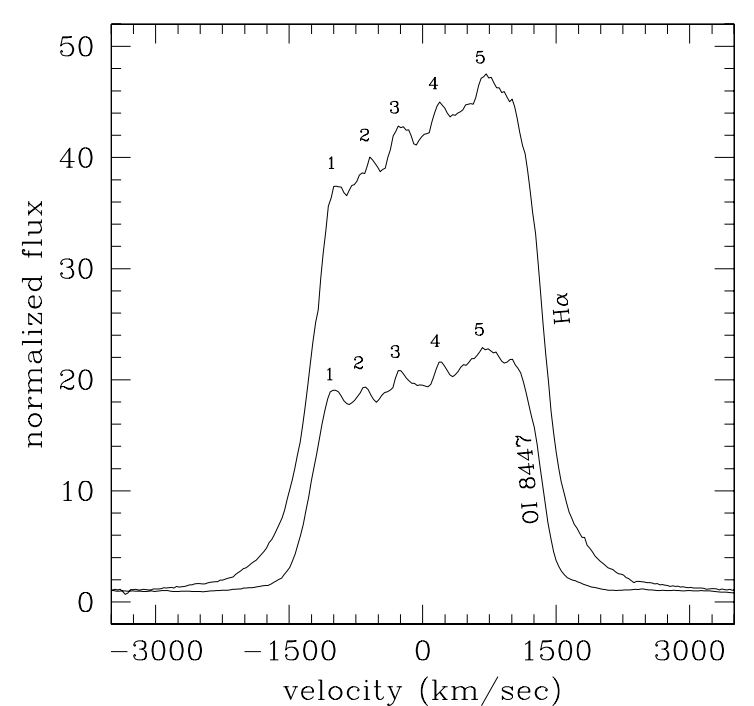

Fig. 2. Resolved $\mathrm{H} \alpha$ and OI $8446 \AA$ emission profiles of Nova Scuti 2005 N.2 observed on 2005 Oct. 27.74 UT. The continuum is normalized to 1.0. The numbers identify the ripples in the profiles whose radial velocity is given in Table 2 .

on Oct. 12.0 (UT) at $V=9.8 \mathrm{mag}$, so for sake of discussion we adopt this in the following.

The nova decline times are $t_{2}=3$ and $t_{3}=6$ days, making it one of the fastest known novae. Among the very few known faster classical novae, there are Nova Her $1991\left(t_{2}=1.2\right.$ and $t_{3}=$ 3.2 days) and Nova Ser 1983 ( $t_{2}=2$ and $t_{3}=5$ days). Using the relation between $t_{2}$ and the outburst amplitude of Warner (1995), the nova progenitor should have shined at $V \geq 23$, so it is no surprise that it does not show up on DSS1 or DSS2 plates. The color of the nova remained stable at $B-V=1.3$ for the first three weeks (cf. bottom panel of Fig. 3), which suggests a reddening amounting to $E_{B-V}=+1.3$ after adopting an intrinsic $(B-V)_{0}=0.0$ at time $t_{2}$ from Warner (1995). This agrees with the Neckel \& Klare (1980) extinction maps that indicate $E_{B-V} \geq 1$ along the line of sight to the nova for any reasonable distance estimate. The equivalent width of the diffuse interstellar band at $6614 \AA$ (barely noticeable at the compressed scale of Fig. 2 on the red wing of the $\mathrm{H} \alpha$ profile) is $0.16( \pm 0.02) \AA$, much larger than the
Table 1. $B V I_{\mathrm{C}}$ CCD photometry of Nova Scuti 2005 N.2. $\mathrm{MJD}_{\odot}=$ heliocentric JD - 2450000 .

\begin{tabular}{crcccccc}
\hline \hline MJD $_{\odot}$ & Date & $B$ & $\sigma_{B}$ & $V$ & $\sigma_{V}$ & $I_{C}$ & $\sigma_{l}$ \\
\hline 3658.27 & Oct. 14.77 & 12.91 & 0.08 & 11.64 & 0.04 & 8.69 & 0.03 \\
3660.28 & 16.78 & 13.74 & 0.09 & 12.48 & 0.05 & 9.30 & 0.02 \\
3666.27 & 22.77 & & & 13.55 & 0.08 & & \\
3672.25 & 28.75 & & & & & 10.93 & 0.02 \\
3677.24 & Nov. 02.74 & & & 14.60 & 0.10 & & \\
3678.23 & 03.73 & & & 14.97 & 0.09 & 11.42 & 0.03 \\
\hline
\end{tabular}

$0.04 \AA$ observed by Herbing (1995) in HD 183143, which is similarly reddened by $E_{B-V}=+1.3$, thus confirming a high reddening affecting Nova Sct 2005 N2, probably even larger than $E_{B-V}=+1.3$.

Using the Cohen (1988) relation between absolute magnitude and $t_{2}$ rate of decline, the nova absolute magnitude is $M_{\mathrm{V}}=$ $\sim-9.5$ (an identical value is found using the Schmidt (1957) relation for $t_{3}$ ), which corresponds to a distance of $11 \mathrm{kpc}$, and a height above the Galactic plane $z \sim 0.6 \mathrm{kpc}$ for $E_{B-V}=+1.3$ and $V_{\max }=9.8 \mathrm{mag}$. Propagating the uncertainties involved with absolute and observed magnitude at maximum and with reddening, the error budget on distance and $z$ is $33 \%$, allowing an association of the nova with the Galactic bulge.

The drop in magnitude indicated by the last photometric point in Fig. 3 suggests that the nova was at that time entering a dust condensation episode in its ejecta or that it was beginning to experience the oscillations sometimes seen during the transition phase of fast novae. The color at that time $V-I_{C}=+3.6$ was significantly redder than the stable mean value $\left\langle V-I_{\mathrm{C}}\right\rangle \sim$ +3.0 characterizing the previous part of the lightcurve. However, this is not a conclusive argument in favor of a dust condensation episode because color changes have sometimes been observed to accompany oscillations, too. Quite unfortunately, bad weather and conjunction with the Sun prevented us from extending the observations in time to properly cover this interesting phase of the lightcurve.

\section{Spectroscopy}

Integrating the $B$ and $V$ magnitudes on the spectrum of Fig. 1 using Buser (1978) transmission profiles provides $B=$ $15.36( \pm 0.05)$ and $V=14.04( \pm 0.04) \mathrm{mag}$, which fit the photometric evolution in Fig. 3 well (where they are represented by 


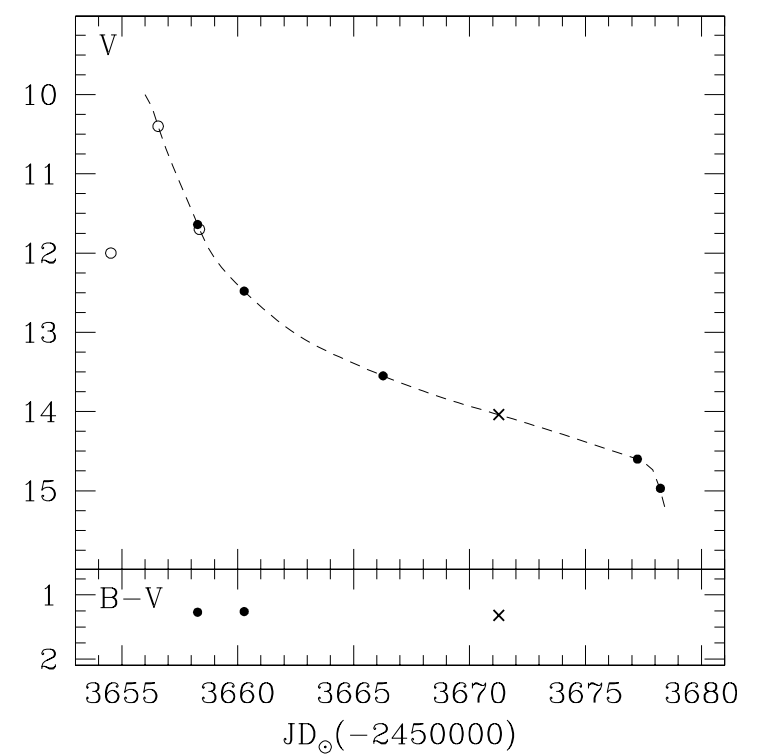

Fig. 3. The photometric evolution of Nova Scuti 2005 N.2. The dots mark our CCD observations in Table 1, the crosses correspond to $B$, $V$ band integration over the absolutely fluxed spectrum of Fig. 1, and the open circles are values published in IAUC 8617. The dashed line is hand drawn.

Table 2. Radial velocity of the ripples numbered on the $\mathrm{H} \alpha$ and OI $8447 \AA$ A emission line profiles of Fig. 2.

\begin{tabular}{lccccc}
\hline \hline & \multicolumn{5}{c}{ Component velocity $\left(\mathrm{km} \mathrm{s}^{-1}\right)$} \\
\cline { 2 - 6 } & 1 & 2 & 3 & 4 & 5 \\
$\mathrm{H} \alpha$ & -970 & -595 & -260 & +180 & +705 \\
$\mathrm{OI} 8446 \AA$ & -990 & -655 & -250 & +200 & +695 \\
\hline
\end{tabular}

the cross symbol), thus enforcing confidence in the accuracy of the calibration into absolute fluxes of the spectrum.

The spectrum in Fig. 1 shows that the nova had not yet entered the nebular phase at the time of observation (+16 days from maximum). The spectrum lacks significant FeII lines and is instead rich in $\mathrm{He}$ and $\mathrm{N}$ lines, allowing us to associate Nova Scuti 2005 N.2 with the He/N class defined by Williams (1992), which is consistent with the very fast speed class of the nova. It is worth noticing that Della Valle \& Livio (1998) found a typical scale height of $\mathrm{He} / \mathrm{N}$ novae above the Galactic plane of $\leq 100 \mathrm{pc}$, significantly less than the $z \sim 0.6 \pm 0.2 \mathrm{kpc}$ we have derived above for this nova. The spectrum in Fig. 1 bears some resemblance to the spectrum of Nova LMC 1990 N.1 presented by Williams et al. (1991), which at later stages evolved into a Neon nova.

The high resolution profiles of $\mathrm{H} \alpha$ and OI $8446 \AA$ in Fig. 2 display a width at half intensity of $2645( \pm 15)$ and $2590( \pm 15) \mathrm{km} \mathrm{s}^{-1}$, respectively, within the observed spread of the McLaughlin (1960) relations between expansion velocity and $t_{2}, t_{3}$ decline rates. These velocities are about $10 \%$ slower than found by Fujii (2005) for observations secured 11 days earlier, in agreement with the expected velocity decrease with time (e.g. Warner 1989).

Both the profiles in Fig. 2 display a series of ripples. Their radial velocities are given in Table 2 . There is good correspondence in the radial velocity of the same ripple observed in the two distinct profiles, supporting a real kinematic identity. Such ripples can be ascribed to large, distinct blobs of material ejected by the nova at different angles with respect to the line of sight (beautifully visible in the HST images of Nova Cyg 1992 and T Pyx). Alternatively, they can be caused by projection effects of equatorial and polar rings of enhanced brightness in the expanding ejecta as shown in the atlas of computed lines profiles by Gill \& O’Brien (1999).

\section{References}

Buser, R. 1978, A\&A, 62, 411

Cohen, J. G. 1988, ASP Conf. Ser., 4, 114

della Valle, M., \& Livio, M. 1998, ApJ, 506, 818

Das, R. K., Ashok, N. M., \& Banerjee, D. P. K. 2005, IAUC, 8617

Fujii, M. 2005, IAUC, 8617

Gill, C. D., \& O'Brien, T. J. 1999, MNRAS, 307, 677

Herbig, G. H. 1995, ARA\&A, 33, 19

Haseda, K. 2005, IAUC, 8617

McLaughlin, D. B. 1960, in Stellar Atmospheres, ed. J. L. Greenstein (Univ. Chicago Press), 585

Pojmanski, G. 2005, IAUC, 8617

Puckett, T. 2005, IAUC, 8617

Schmidt, T. 1957, ZA, 41, 182

Warner, B. 1989, in Classical Novae, ed. M. F. Bode, \& A. Evans (Wiley \& Sons publishers), 1

Warner, B. 1995, Cataclysmic Variable Stars (Cambridge Univ. Press)

Williams, R. E. 1992, AJ, 104, 725

Williams, R. E., Hamuy, M., Phillips, M. M., et al. 1991, ApJ, 376, 721 